\title{
Zeno dynamics and constraints
}

\author{
P. Facchi $\uparrow \S$, G. Marmo $\ddagger$, S. Pascazio $\dagger$, A. Scardicchio $\uparrow$ and \\ E.C.G. Sudarshan $\sharp$ \\ $\dagger$ Dipartimento di Fisica, Università di Bari \\ and Istituto Nazionale di Fisica Nucleare, Sezione di Bari, I-70126 Bari, Italy \\ $\ddagger$ Dipartimento di Scienze Fisiche, Università Federico II di Napoli \\ and Istituto Nazionale di Fisica Nucleare, Sezione di Napoli, I- 80126 Napoli, Italy \\ I Center for Theoretical Physics, Massachusetts Institute of Technology, Cambridge, \\ MA 02139, USA \\ $\sharp$ Physics Department, University of Texas at Austin Texas 78712, USA
}

\begin{abstract}
We investigate some examples of quantum Zeno dynamics, when a system undergoes very frequent (projective) measurements that ascertain whether it is within a given spatial region. In agreement with previously obtained results, the evolution is found to be unitary and the generator of the Zeno dynamics is the Hamiltonian with hard-wall (Dirichlet) boundary conditions. By using a new approach to this problem, this result is found to be valid in an arbitrary $N$-dimensional compact domain. We then propose some preliminary ideas concerning the algebra of observables in the projected region and finally look at the case of a projection onto a lower dimensional space: in such a situation the Zeno ansatz turns out to be a procedure to impose constraints.
\end{abstract}

$\S$ To whom correspondence should be addressed (paolo.facchi@ba.infn.it) 


\section{Introduction}

Very frequent measurement can slow the time evolution of quantum mechanical systems. This is, in a few words, the quantum Zeno effect (QZE), by which transitions to states different from the initial one are gradually suppressed as the measurement frequency $N$ becomes very large [1, 2] (for a review, see [3]). There are, however, two important issues that deserve attention: firstly, for a general (incomplete and nonselective [4]) measurement, represented by a complete set of projections onto multidimensional subspaces (rather than a single-dimensional one, as in the usual formulation of the QZE, by which the measurement ascertains whether the system is still in its initial, pure state), the quantum system may - and indeed does - evolve away from its initial state, although it remains in the subspace defined by the measurement (and represented by a multidimensional projection operator) [5, 6]. This leads to the formation of the "Zeno subspaces" 7]. Secondly, if the measurement is not very frequent, the quantum evolution yields the so-called "inverse" or "anti" Zeno effect, by which transitions away from the initial state (or in general out of the relevant subspaces) are accelerated 8 .

Both the Zeno and inverse Zeno phenomena have been experimentally observed during the last few years [9, 10, 11, 12] (but see [13, for previous analyses of experimental data on nuclear hadronic cascades). The first experiment was done with an oscillating system [9], according to an interesting proposal by Cook 14, and was widely debated 15. In a recent beautiful set of experiments, performed by Raizen's group, first the initial quadratic and non-Markovian Zeno region was observed [10, then both the quantum Zeno and inverse Zeno effects were proved for bona fide unstable system (probability leakage out of an optical potential) [11].

In this article we shall mainly analyze the first issue, investigating the features

of the Zeno (sub)dynamics in the relevant subspace. This and related problems were contemplated in the seminal formulation of the QZE [2], where it was proved that the dynamics is governed by a semigroup. The details of the dynamics had interesting and challenging mathematical aspects, that were independently investigated by other authors [16, 17]. As a matter of fact, some mathematical issues are still unresolved nowadays. One of the most intruiguing features of the original paper [2] is that some delicate operator properties were postulated on physical grounds; curiously, these postulates are always found to be valid in concrete examples, even nontrivial ones.

For a wide class of measurements, namely those represented by spatial projections, one can prove that the system evolves unitarily in a proper subspace of the total Hilbert space, the generator of the dynamics being the Hamiltonian with Dirichlet boundary conditions on the region associated with the spatial projection [5, 6]. This finding motivated further interesting studies on this topic [18, 19, 20, 21]. In particular, Exner and Ichinose 21] analyzed this result in a rigorous framework, under the nontrivial (and interesting) assumption that the original Hamiltonian be lower bounded and the Zeno Hamiltonian densely defined in the Hilbert space. The aim of this article is to further elaborate on these issues. We will first explicitly work out some examples- 
essentially the free case in two and three dimensions, with projections onto regular domains - and introduce a novel calculation technique, giving a constructive proof of the Zeno Hamiltonian. We then extend this result to a general spatial projection in $N$ dimensions.

We shall prove that the Dirichlet boundary conditions are a consequence of the Zeno procedure (different proofs can be given, at different levels of generality and mathematical rigor, see [16, 5, 6, 21]), by exploring an interesting method of calculation, based on asymptotic techniques, that yields a stationary Schrödinger equation with the appropriate (Dirichlet) boundary conditions for its eigenfunctions. In Sec. 2 we set up the general framework and introduce notation. In Sec. 3 the projection domain is a rectangle in the plane. In Sec. 4 it is an annulus in the plane. In Sec. 5 we look at a spherical shell in $\mathbb{R}^{3}$. In Sec. [6] we generalize to regular domains in $\mathbb{R}^{N}$ and in Sec. [7] we briefly discuss the Zeno dynamics in the Heisenberg picture as well as the features of the algebra of observables in the projected domain. In Sec. 8 we look at a different case, when the system is projected onto a domain of lower dimensionality: we shall only look at some examples and shall not attempt to generalize. One can say that in this case the Zeno ansatz yields a procedure to impose a constraint. The ideas we propose in these last two sections are somewhat embryonic and can be considered as plans for future developments. In Sec. 9 we comment on future perspectives and applications.

\section{Zeno subdynamics}

Consider a free particle in $N$-dimensions with the Hamiltonian

$$
H=\frac{\boldsymbol{p}^{2}}{2 M}=-\frac{\hbar^{2} \triangle}{2 M}, \quad U(t)=e^{-i H t / \hbar}
$$

acting on $\psi \in L^{2}\left(\mathbb{R}^{N}\right)$. Given a compact domain $D \subset \mathbb{R}^{N}$ with a nonempty interior and a regular boundary, consider the projection operator

$$
P=\chi_{D}(\boldsymbol{x})=\int_{D} d^{N} \boldsymbol{x}|\boldsymbol{x}\rangle\langle\boldsymbol{x}|, \quad P \psi(\boldsymbol{x})=\chi_{D}(\boldsymbol{x}) \psi(\boldsymbol{x}),
$$

where $\chi_{D}(\boldsymbol{x})$ is the characteristic function of the domain $D$, and thought of as an operator, along with its complement $Q=1-P=1-\chi_{D}(\boldsymbol{x})$, decomposes the space $L^{2}\left(\mathbb{R}^{N}\right)$ into orthogonal subspaces. The Zeno subdynamics evolution operator is given by the limit

$$
U_{Z}(t)=\lim _{N \rightarrow \infty}(G(t / N))^{N}
$$

where the (nonunitary) evolution

$$
G(\tau)=P U(\tau) P
$$

represents a single step (projection-evolution-projection) Zeno process.

Under rather general hypotheses the limit (3) can be proved to exist [16, 2, 15, 6, 21] and yields a unitary evolution group in a proper subspace of $L^{2}(D)$. One gets

$$
U_{Z}(t)=P \exp \left(-i H_{Z} t / \hbar\right)
$$


where the generator of the dynamics is the Zeno Hamiltonian

$$
H_{Z}=-\frac{\hbar^{2} \triangle_{D}}{2 M}
$$

defined in the domain

$$
D\left(H_{Z}\right)=\left\{\psi \in L^{2}(D) \mid \triangle \psi \in L^{2}(D), \psi(\partial D)=0\right\},
$$

$\partial D$ being the boundary of $D$ (hard-wall or Dirichlet boundary conditions).

We will focus on this problem by looking for the eigenbasis $\{|\boldsymbol{n}\rangle\}$ of $U_{Z}(t)$ in the subspace $P L^{2}\left(\mathbb{R}^{N}\right) \simeq L^{2}(D)$ such that

$$
\left\langle\boldsymbol{n}\left|U_{Z}(t)\right| \boldsymbol{m}\right\rangle=\lim _{N \rightarrow \infty}\left\langle\boldsymbol{n}\left|G(t / N)^{N}\right| \boldsymbol{m}\right\rangle=\left\langle\boldsymbol{n}\left|e^{-i H_{Z} t / \hbar}\right| \boldsymbol{m}\right\rangle=\delta_{\boldsymbol{m}, \boldsymbol{n}} e^{-i E_{\boldsymbol{n}} t / \hbar} \cdot(8)
$$

In order to find this basis consider an arbitrary orthonormal complete set of functions in $L^{2}(D)$

$$
\Psi_{\boldsymbol{n}}(\boldsymbol{x})=\langle\boldsymbol{x} \mid \boldsymbol{n}\rangle
$$

and take the matrix elements of the single-step operator (4)

$$
G_{\boldsymbol{m}, \boldsymbol{n}}(t)=\langle\boldsymbol{m}|G(t)| \boldsymbol{n}\rangle=\operatorname{Tr}[G(t)|\boldsymbol{n}\rangle\langle\boldsymbol{m}|] .
$$

If the matrix elements of the single-step operator behave like

$$
G_{\boldsymbol{m}, \boldsymbol{n}}(t)=\delta_{\boldsymbol{m}, \boldsymbol{n}}\left(1-i \frac{E_{\boldsymbol{n}} t}{\hbar}\right)+R_{\boldsymbol{m}, \boldsymbol{n}}(t)
$$

where for $t \rightarrow 0$

$$
R_{\boldsymbol{m}, \boldsymbol{n}}(t)=o(t),
$$

then, under the assumption of uniform convergence of the infinite sums stemming from the insertion of $N-1$ resolutions of the identity in (3), one obtains:

$$
\begin{aligned}
G_{\boldsymbol{m}, \boldsymbol{n}}^{Z}(t) & \equiv\left\langle\boldsymbol{m}\left|U_{Z}(t)\right| \boldsymbol{n}\right\rangle \\
& =\lim _{N \rightarrow \infty} \sum_{\boldsymbol{n}_{1}, \ldots, \boldsymbol{n}_{N-1}} G_{\boldsymbol{m}, \boldsymbol{n}_{1}}(t / N) G_{\boldsymbol{n}_{1}, \boldsymbol{n}_{2}}(t / N) \cdots G_{\boldsymbol{n}_{N-1}, \boldsymbol{n}}(t / N) \\
& =\delta_{\boldsymbol{m}, \boldsymbol{n}} \exp \left(-i \frac{E_{\boldsymbol{n}} t}{\hbar}\right) .
\end{aligned}
$$

The basis $\{|\boldsymbol{n}\rangle\}$ is thus the eigenbasis of $H_{Z}$ belonging to the eigenvalues $E_{\boldsymbol{n}}$ :

$$
H_{Z} \Psi_{\boldsymbol{n}}(\boldsymbol{x})=E_{\boldsymbol{n}} \Psi_{\boldsymbol{n}}(\boldsymbol{x}) .
$$

Notice that when we apply $U(t / N)$ to the relevant subspace $P L^{2}\left(\mathbb{R}^{N}\right)$, the transformed space need not be orthogonal anymore to $Q L^{2}\left(\mathbb{R}^{N}\right)$, where $Q=1-P$, and the $t / N$ dependence of the scalar product of two vectors in these two subspaces is given by

$$
Q U(t / N) P=O(t / N) \text {. }
$$

It has been shown that Eq. (11) implies Dirichlet boundary conditions for the states $\Psi_{\boldsymbol{n}}(\boldsymbol{x})$ (the "Zeno eigenbasis") [6]. The proof, based on asymptotic techniques, yields the propagator in an appropriately chosen basis of eigenfunctions. In the following sections we shall introduce a novel approach: by using asymptotic analysis and the 
path integral representation of the matrix element (10), we will obtain a stationary Schrödinger equation and a set of boundary conditions for its eigenfunctions. This will enable us to define the induced Zeno Hamiltonian $H_{Z}$ and its spectrum. The advantage of the present approach, as compared to the previous one [6], lies in the fact that one can derive a Schrödinger equation with Dirichlet boundary conditions in the projected (Zeno) subspace. Moreover, by examining some examples of multiple connected domains, we will show how the Zeno dynamics induces constraints that inherit the topological properties of the parent space.

\section{Rectangle}

We start off with one of the simplest examples and introduce the procedure. Consider a rectangle in the plane, $D=[0, a] \times[0, b] \subset \mathbb{R}^{2}$. In this case the projection (2) reads

$$
P=\chi_{[0, a]}(x) \chi_{[0, b]}(y)=\int_{0}^{a} d x \int_{0}^{b} d y|x y\rangle\langle x y|
$$

and the Hamiltonian (1) is

$$
H=\frac{p_{x}^{2}+p_{y}^{2}}{2 M}=-\frac{\hbar^{2}}{2 M}\left(\partial_{x}^{2}+\partial_{y}^{2}\right) .
$$

The Zeno Hamiltonian, engendering the Zeno subdynamics, is formally given by (66)-(7) and represents a free particle in the box $D=[0, a] \times[0, b]$ with Dirichlet boundary conditions

$$
\begin{aligned}
H_{Z}= & -\frac{\hbar^{2}}{2 M}\left(\partial_{x}^{2}+\partial_{y}^{2}\right) \\
& \psi(0, y)=\psi(a, y)=0, \quad \psi(x, 0)=\psi(x, b)=0
\end{aligned}
$$

The eigenfunctions and eigenvalues are well known

$$
\begin{aligned}
& \Psi_{n m}(x, y)=\sqrt{\frac{2}{a}} \sin \left(\frac{n \pi}{a} x\right) \sqrt{\frac{2}{b}} \sin \left(\frac{n \pi}{b} y\right), \\
& E_{n m}=\frac{\hbar^{2} \pi^{2}}{2 M}\left(\frac{n^{2}}{a^{2}}+\frac{m^{2}}{b^{2}}\right) .
\end{aligned}
$$

Let us look in detail at the derivation of the Zeno subdynamics (18)-(21) in this particular case. As explained in Sec. 2, the eigenbasis of the Zeno Hamiltonian $H_{Z}$ in $L^{2}(D)$,

$$
\Psi_{n m}(x, y)=\langle x, y \mid n m\rangle
$$

must satisfy condition (11):

$$
G_{n^{\prime} m^{\prime}, n m}(t)=\delta_{n^{\prime} n} \delta_{m^{\prime} m}\left(1-i \frac{E_{n m} t}{\hbar}\right)+o(t),
$$

where

$$
G_{n^{\prime} m^{\prime}, n m}(t)=\left\langle n^{\prime} m^{\prime}|G(t)| n m\right\rangle .
$$

are the matrix elements (10) of the single-step evolution operator. 
This can be proved by direct inspection: one gets

$$
\begin{aligned}
G_{n^{\prime} m^{\prime}, n m}(t)= & \int_{0}^{a} d x \int_{0}^{b} d y \int_{0}^{a} d x^{\prime} \int_{0}^{b} d y^{\prime}\left(\frac{M}{2 \pi i \hbar t}\right) e^{i \frac{M\left(\left(x^{\prime}-x\right)^{2}+\left(y^{\prime}-y\right)^{2}\right)}{2 \hbar t}} \\
& \times \Psi_{n^{\prime} m^{\prime}}^{*}(x, y) \Psi_{n m}\left(x^{\prime}, y^{\prime}\right)
\end{aligned}
$$

and by substituting $\xi=x^{\prime}-x$ and $\eta=y^{\prime}-y$

$$
\begin{aligned}
G_{n^{\prime} m^{\prime}, n m}(t)= & \int_{0}^{a} d x \int_{0}^{b} d y \int_{-x}^{a-x} d \xi \int_{-y}^{b-y} d \eta\left(\frac{M}{2 \pi i \hbar t}\right) e^{i \frac{M\left(\xi^{2}+\eta^{2}\right)}{2 \hbar t}} \\
& \times \Psi_{n^{\prime} m^{\prime}}^{*}(x, y) \Psi_{n m}(x+\xi, y+\eta) .
\end{aligned}
$$

With the natural choice $\Psi_{n m}(x, y)=\psi_{n}(x) \phi_{m}(y)$ this yields the product of two quantities

$$
\begin{aligned}
G_{n^{\prime} m^{\prime}, n m}(t)= & G_{n^{\prime} n}(t) G_{m^{\prime} m}(t) \\
= & \int_{0}^{a} d x \int_{-x}^{a-x} d \xi\left(\frac{M}{2 \pi i \hbar t}\right)^{1 / 2} e^{i \frac{M \xi^{2}}{2 \hbar t}} \psi_{n^{\prime}}^{*}(x) \psi_{n}(x+\xi) \\
& \times \int_{0}^{b} d y^{\prime} \int_{-y}^{b-y} d \eta\left(\frac{M}{2 \pi i \hbar t}\right)^{1 / 2} e^{i \frac{M \eta^{2}}{2 \hbar t}} \phi_{m^{\prime}}^{*}(y) \phi_{m}(y+\eta)
\end{aligned}
$$

and accordingly $E_{n m}=E_{n}+E_{m}$. Consider the first quantity $G_{n^{\prime} n}$ and the integral over $\xi$. In the small- $t$ limit there are contributions from the boundary points $\xi=-x$ and $\xi=a-x$ and from the stationary point $\xi=0$

$$
G_{n^{\prime} n}=\int_{0}^{a} d x \psi_{n^{\prime}}^{*}(x)[\text { bound }+ \text { stat }]
$$

where

$$
\begin{aligned}
\text { bound }= & \left.\left(\frac{M}{2 \pi i \hbar t}\right)^{1 / 2} \frac{\hbar t}{i M \xi} \psi_{n}(x+\xi) e^{i \frac{M \xi^{2}}{2 \hbar t}}\right|_{\xi=-x} ^{\xi=a-x}+O\left(t^{3 / 2}\right) \\
& =\sqrt{\frac{\hbar t}{-2 \pi i M}}\left(\frac{e^{i M(x-a)^{2} / 2 \hbar t}}{x-a} \psi_{n}(a)-\frac{e^{i M x^{2} / 2 \hbar t}}{-x} \psi_{n}(0)\right)+O\left(t^{3 / 2}\right),
\end{aligned}
$$

while $(\lambda=M / 2 \hbar t)$

$$
\begin{aligned}
\text { stat } & =\int_{-\infty}^{\infty} d \xi\left(\psi_{n}(x)+\psi_{n}^{\prime}(x) \xi+\frac{1}{2 !} \psi_{n}^{\prime \prime}(x) \xi^{2}+O\left(\xi^{3}\right)\right) \sqrt{\frac{\lambda}{\pi i}} e^{i \lambda \xi^{2}} \\
& =\psi_{n}(x)+i \frac{t \hbar}{2 M} \psi_{n}^{\prime \prime}(x)+O\left(t^{2}\right) .
\end{aligned}
$$

In order to obtain (23) one must require that (remember that $E_{n m}=E_{n}+E_{m}$ )

$$
\text { bound }=O\left(t^{3 / 2}\right) \quad \text { and } \quad-\frac{\hbar}{2 M} \psi_{n}^{\prime \prime}(x)=\frac{E_{n}}{\hbar} \psi_{n}(x),
$$

which translates into

$$
-\frac{\hbar^{2}}{2 M} \partial_{x}^{2} \psi_{n}(x)=E_{n} \psi_{n}(x), \quad \text { with } \quad \psi_{n}(0)=\psi_{n}(a)=0
$$


so that for $G_{n^{\prime} n}$ one obtains

$$
G_{n^{\prime} n}(t)=\left(1-i \frac{E_{n} t}{\hbar}\right) \delta_{n^{\prime} n}+O\left(t^{3 / 2}\right)
$$

and analogously for $G_{m^{\prime} m}$, so that

$$
G_{n^{\prime} m^{\prime}, n m}(t)=\left(1-i \frac{E_{n} t}{\hbar}-i \frac{E_{m} t}{\hbar}\right) \delta_{n^{\prime} n} \delta_{m^{\prime} m}+O\left(t^{3 / 2}\right),
$$

which has exactly the form (23). By Eq. (32) and its analog for $\phi_{m}(y)$, the eigenfunctions $\Psi_{n m}(x, y)=\psi_{n}(x) \phi_{m}(y)$ of $H_{Z}$ satisfy

$$
-\frac{\hbar^{2}}{2 M}\left(\partial_{x}^{2}+\partial_{y}^{2}\right) \Psi_{n, m}(x, y)=\left(E_{n}+E_{m}\right) \Psi_{n, m}(x, y)
$$

with Dirichlet boundary conditions. They are therefore given by (20). The Zeno Hamiltonian is therefore (18)-(19).

This derivation, although it yields the desired (and correct) result, is not rigorous. In particular, it does not tackle the delicate problem of understanding the convergence properties of the asymptotic expansion at the intersection of the $(x, y)$ and $\left(x^{\prime}, y^{\prime}\right)$ boundaries in Eq. (25) [this is apparent if one looks at the denominators of the far right hand side of Eq. (29)]. A similar approach will be adopted in the next sections. A more rigorous proof can be given, but will not be presented here.

\section{Annulus}

Consider now a circular annulus (or ring) of width $\delta r=r_{2}-r_{1}$ on the plane, defining the domain $D=\left\{(x, y) \mid r_{1}^{2} \leq x^{2}+y^{2} \leq r_{2}^{2}\right\}$. The projection on $D$ reads

$$
\begin{aligned}
& P=\chi_{\left[r_{1}, r_{2}\right]}(r)=\int_{D} d x d y|x y\rangle\left\langle x y\left|=\int_{r_{1}}^{r_{2}} d r r \int_{0}^{2 \pi} d \theta\right| r \theta\right\rangle\langle r \theta| . \\
& r_{2}-r_{1} \equiv \delta r>0 .
\end{aligned}
$$

The Zeno Hamiltonian, engendering the Zeno subdynamics, is given by (6) and represents a free particle on $D$ with Dirichlet boundary condition

$$
\begin{gathered}
H_{Z}=-\frac{\hbar^{2}}{2 M}\left(\partial_{x}^{2}+\partial_{y}^{2}\right)=-\frac{\hbar^{2}}{2 M}\left(\frac{1}{r} \partial_{r}\left(r \partial_{r}\right)+\frac{1}{r^{2}} \partial_{\theta}^{2}\right), \\
\psi\left(r_{1}, \theta\right)=\psi\left(r_{2}, \theta\right)=0 .
\end{gathered}
$$

As is well known, by writing the eigenfunctions of $H_{Z}$ as $\Psi_{n l}(r, \theta)=\psi_{n l}(r) \phi_{l}(\theta)$, the angular functions are given by

$$
\phi_{l}(\theta)=\frac{1}{\sqrt{2 \pi}} \exp (i l \theta), \quad \text { with } \quad l=0, \pm 1, \pm 2, \ldots,
$$

while the radial part of the eigenvalue equation reads

$$
\begin{gathered}
-\frac{\hbar^{2}}{2 M} \frac{1}{r} \partial_{r}\left(r \partial_{r}\right) \psi_{n l}(r)+\frac{\hbar^{2} l^{2}}{2 M r^{2}} \psi_{n l}(r)=E_{n l} \psi_{n l}(r) \\
\psi_{n l}\left(r_{1}\right)=\psi_{n l}\left(r_{2}\right)=0
\end{gathered}
$$


and can be solved in terms of Bessel functions.

Let us look in detail at the derivation of the Zeno subdynamics (38)-(42) in this particular case. As explained in Sec. 2, the eigenbasis of the Zeno Hamiltonian $H_{Z}$ in $L^{2}(D)$,

$$
\Psi_{n l}(r, \theta)=\langle r, \theta \mid n l\rangle
$$

must satisfy condition (11), that is

$$
G_{n^{\prime} l^{\prime}, n l}(t)=\delta_{n^{\prime} n} \delta_{l^{\prime} l}\left(1-i \frac{E_{n l} t}{\hbar}\right)+o(t),
$$

where

$$
G_{n^{\prime} l^{\prime}, n l}(t)=\left\langle n^{\prime} l^{\prime}|G(t)| n l\right\rangle
$$

are the matrix elements (10) of the single-step evolution operator.

By writing $\Psi_{n l}(r, \theta)=\psi_{n l}(r) \phi_{l}(\theta)$, we get

$$
\begin{aligned}
G_{n^{\prime} l^{\prime}, n l}(t)= & \int_{r_{1}}^{r_{2}} r d r \int_{r_{1}}^{r_{2}} r^{\prime} d r^{\prime} \int_{0}^{2 \pi} d \theta \int_{0}^{2 \pi} d \theta^{\prime} \\
& \times \psi_{n l}(r) \phi_{l}(\theta) \psi_{n^{\prime} l^{\prime}}^{*}\left(r^{\prime}\right) \phi_{l^{\prime}}^{*}\left(\theta^{\prime}\right)\left(\frac{M}{2 \pi i \hbar t}\right) e^{i \frac{M d^{2}}{2 \hbar t}}
\end{aligned}
$$

where $d$ is the distance between the points $(r, \theta)$ and $\left(r^{\prime}, \theta^{\prime}\right)$

$$
d^{2}=r^{\prime 2}+r^{2}-2 r^{\prime} r \cos \left(\theta^{\prime}-\theta\right)=\left(r^{\prime}-r\right)^{2}+2 r^{\prime} r\left(1-\cos \left(\theta^{\prime}-\theta\right)\right) .
$$

Let us look first at the $\theta$ integrals. Changing again to $\eta=\theta-\theta^{\prime}$ and dropping the prime one gets

$$
\begin{aligned}
G_{n^{\prime} l^{\prime}, n l}(t)= & \int_{r_{1}}^{r_{2}} r d r \int_{r_{1}}^{r_{2}} r^{\prime} d r^{\prime} \psi_{n l}(r) \psi_{n^{\prime} l^{\prime}}^{*}\left(r^{\prime}\right)\left(\frac{M}{2 \pi i \hbar t}\right)^{1 / 2} e^{i \frac{M\left(r^{\prime}-r\right)^{2}}{2 \hbar t}} \\
& \times \int_{0}^{2 \pi} d \theta \int_{-\theta}^{2 \pi-\theta} d \eta \phi_{l}(\theta+\eta) \phi_{l^{\prime}}^{*}(\theta)\left(\frac{M}{2 \pi i \hbar t}\right)^{1 / 2} e^{i \frac{M r^{\prime} r}{\hbar t}(1-\cos \eta) .}
\end{aligned}
$$

Consider the integral over $\eta$ (at fixed $r^{\prime}$ and $r$ ). In the limit $t \rightarrow 0$ the boundary contribution reads $\left(z=M r^{\prime} r / \hbar t\right)$

$$
\text { bound }=\frac{1}{\sqrt{r^{\prime} r}} \frac{-i}{\sqrt{2 \pi i z \sin ^{2} \theta}} e^{i z(1-\cos (\theta))}\left[\phi_{l}(2 \pi)-\phi_{l}(0)\right]+O\left(t^{3 / 2}\right) .
$$

In order that $O(\sqrt{t})$ vanishes and (44) is satisfied, one must require the periodicity

$$
\phi_{l}(0)=\phi_{l}(2 \pi) \text {. }
$$

The difference with the preceding case is given by the periodicity of the Green function.

However, we now have two stationary points in the $\eta$ integral. One is $\eta=0$ and the other is $\eta=\pi$ for $\theta<\pi$, or $\eta=-\pi$ for $\theta>\pi$. These represent the minimum and maximum of the distance between two points, one fixed on the circle $r^{\prime}=$ const. and the other one located on the circle $r=$ const. at an angle $\eta$. One should get (at least) two points of stationary phase each time one constrains the dynamics on a closed 
(iper)surface. Both contributions must be taken into account. The only difference with the previous case is that one must consider also $\eta^{4}$ terms arising from the cosine in the integral

$$
\begin{aligned}
\operatorname{stat}_{0}= & \frac{1}{\sqrt{r^{\prime} r}} \int d \eta \sqrt{\frac{z}{2 \pi i}}\left[\phi_{l}(\theta)+\frac{1}{2 !} \phi_{l}^{\prime \prime}(\theta) \eta^{2}-i \frac{z}{4 !} \eta^{4} \phi_{l}(\theta)\right] e^{i z \eta^{2} / 2} \\
\text { stat }_{ \pm \pi}= & \frac{1}{\sqrt{r^{\prime} r}} e^{2 i z} \int d \eta \sqrt{\frac{z}{2 \pi i}}\left[\phi_{l}(\theta \pm \pi)+\frac{1}{2 !} \phi_{l}^{\prime \prime}(\theta \pm \pi) \eta^{2}\right. \\
& \left.+i \frac{z}{4 !} \eta^{4} \phi_{l}(\theta \pm \pi)\right] e^{-i z \eta^{2} / 2}
\end{aligned}
$$

Notice that stat $_{ \pm \pi}$ has a phase $2 z=2 m r^{\prime} r / \hbar t$. This phase changes the term $m\left(r^{\prime}-r\right)^{2} / 2 \hbar t$ of the $r^{\prime}, r$ integrals into a term $m\left(r^{\prime}+r\right)^{2} / 2 \hbar t$. This factor has no more stationary points in the $r^{\prime}, r$ integrals, so that its contribution can be neglected (in the $t \rightarrow 0$ limit). In turn, also the contribution from stat ${ }_{ \pm \pi}$ can be neglected. On the other hand, the stat ${ }_{0}$ contribution is

$$
\text { stat }_{0}=\frac{1}{\sqrt{r^{\prime} r}}\left[\phi_{l}(\theta)+\frac{i \hbar t}{2 M r^{\prime} r} \phi_{l}^{\prime \prime}(\theta)+\frac{i \hbar t}{8 M r^{\prime} r} \phi_{l}(\theta)\right]+O\left(t^{2}\right)
$$

and following the same reasoning as in Sec. 2 (rectangle on the plane) one obtains a differential equation for the eigenfunctions

$$
-\phi_{l}^{\prime \prime}(\theta)=\alpha_{l} \phi_{l}(\theta), \quad \phi(0)=\phi(2 \pi)
$$

which yields $\alpha_{l}=l^{2}$, whence

$$
\int_{0}^{2 \pi} d \theta \phi_{l^{\prime}}^{*}(\theta) \operatorname{stat}_{0}=\delta_{l^{\prime} l} \frac{1}{\sqrt{r^{\prime} r}}\left[1-i t \frac{\hbar}{2 M r^{\prime} r}\left(l^{2}-\frac{1}{4}\right)\right]+O\left(t^{2}\right) .
$$

Therefore the integral over $r^{\prime}, r$ reads

$$
\begin{aligned}
& \int_{r_{1}}^{r_{2}} r d r \int_{r_{1}}^{r_{2}} r^{\prime} d r^{\prime} \sqrt{\frac{M}{2 \pi i \hbar t}} \psi_{n^{\prime} l^{\prime}}^{*}\left(r^{\prime}\right) \psi_{n l}(r) e^{i \frac{M\left(r^{\prime}-r\right)^{2}}{2 \hbar t}} \delta_{l^{\prime} l} \\
& \quad \times \frac{1}{\sqrt{r^{\prime} r}}\left[1-i t \frac{\hbar}{2 M r^{\prime} r}\left(l^{2}-\frac{1}{4}\right)\right] .
\end{aligned}
$$

By inserting $\xi=r-r^{\prime}$ and dropping the prime on $r^{\prime}$ we get

$$
\begin{aligned}
& \int_{r_{1}}^{r_{2}} \sqrt{r} d r \int_{r_{1}-r}^{r_{2}-r} \sqrt{\xi+r} d \xi \sqrt{\frac{M}{2 \pi i \hbar t}} \psi_{n^{\prime} l^{\prime}}^{*}(r) \psi_{n l}(r+\xi) e^{i \frac{M \xi^{2}}{2 \hbar t}} \delta_{l^{\prime} l} \\
& \quad \times\left[1-i t \frac{\hbar}{2 M r(r+\xi)}\left(l^{2}-\frac{1}{4}\right)\right] .
\end{aligned}
$$

By the same reasoning as before one obtains a differential equation and the Dirichlet boundary conditions for the functions $A_{n l}(r)=\sqrt{r} \psi_{n l}(r)$ :

$$
\begin{gathered}
-\frac{\hbar^{2}}{2 M} A_{n l}^{\prime \prime}(r)+\frac{\hbar^{2}}{2 M r^{2}}\left(l^{2}-\frac{1}{4}\right) A_{n l}(r)=E_{n l} A_{n l}(r), \\
A_{n l}\left(r_{1}\right)=A_{n l}\left(r_{2}\right)=0 .
\end{gathered}
$$

In terms of the radial functions $\psi_{n l}$, Eq. (58) becomes just Eq. (41), whence the Zeno Hamiltonian is given by (38)-(39). 
It is interesting to notice that in this case of multiple connectedness the Zeno dynamics yields no Aharonov-Bohm topological phases. In a few words, one might say that the projected dynamics on the annulus "inherits" the topological properties of the initial space $\mathbb{R}^{2}$, and in particular the single valuedness of the wave function. The spatial projections do not introduce any additional "twist" into the system, that could induce a phase.

Two additional quick comments: first, the $r_{1} \rightarrow 0$ limit yields a circle; however, it does not yield the Zeno dynamics on the domain $D=\left\{(x, y) \mid x^{2}+y^{2}<r_{2}^{2}\right\}$, because of the spurious condition $\psi_{n l}(0)=0$, excluding $s$-wave eigenfunctions. This seemingly trivial remark clarifies that taking a limit of the projected domain does not necessarily yield the right Zeno dynamics. Second, the circular ring sector $\left\{(r, \theta) \mid r_{1} \leq r \leq r_{2}, \theta_{1} \leq\right.$ $\left.\theta \leq \theta_{2}\right\}$ can be easily computed and yields the right dynamics and eigenfunctions (Bessel functions $\left.I_{\mu}(r), \mu \in \mathbb{R}\right)[22$.

\section{Spherical shell}

Let us now consider an example in $\mathbb{R}^{3}$. We first observe that the parallelepiped can be easily dealt with by extending the techniques of Sec. 3 . We therefore look at a more interesting situation. Consider a spherical shell in $\mathbb{R}^{3}$ and a domain $D=\left\{(x, y, z) \mid r_{1}^{2} \leq x^{2}+y^{2}+z^{2} \leq r_{2}^{2}\right\}$. The projection on $D$ reads

$$
\begin{aligned}
P=\chi_{\left[r_{1}, r_{2}\right]}(r) & =\int_{D} d x d y d z|x y z\rangle\langle x y z| \\
& =\int_{r_{1}}^{r_{2}} r^{2} d r \int_{0}^{\pi} \sin \theta d \theta \int_{0}^{2 \pi} d \phi|r \theta \phi\rangle\langle r \theta \phi| .
\end{aligned}
$$

The Zeno Hamiltonian, engendering the Zeno subdynamics, is given by (6) and represents a free particle in the spherical shell $D$ with Dirichlet boundary condition

$$
\begin{aligned}
H_{Z}= & -\frac{\hbar^{2}}{2 M}\left(\partial_{x}^{2}+\partial_{y}^{2}+\partial_{y}^{2}\right) \\
=- & \frac{\hbar^{2}}{2 M r^{2}}\left(\partial_{r}\left(r^{2} \partial_{r}\right)+\frac{1}{\sin \theta} \partial_{\theta}\left(\sin \theta \partial_{\theta}\right)+\frac{1}{\sin ^{2} \theta} \partial_{\phi}^{2}\right), \\
& \psi\left(r_{1}, \theta, \phi\right)=\psi\left(r_{2}, \theta, \phi\right)=0 .
\end{aligned}
$$

As is well known, by writing the eigenfunctions of $H_{Z}$ as $\Psi_{n l m}(r, \theta, \phi)=$ $R_{n l}(r) Y_{l m}(\theta) \Phi_{m}(\phi)$, the radial part of the eigenvalue equation reads

$$
\begin{gathered}
-\frac{\hbar^{2}}{2 M} \frac{1}{r^{2}} \partial_{r}\left(r^{2} \partial_{r}\right) R_{n l}(r)+\frac{\hbar^{2}}{2 M} \frac{l(l+1)}{r^{2}} R_{n l}(r)=E_{n l} R_{n l}(r), \\
R_{n l}\left(r_{1}\right)=R_{n l}\left(r_{2}\right)=0
\end{gathered}
$$

and can be solved in terms of spherical Bessel functions.

Let us see how one can obtain $H_{Z}$ in this case. The first steps of the derivation are the same as before. By rewriting the distance $d\left(r^{\prime} \theta^{\prime} \phi^{\prime}, r \theta \phi\right)$ as

$$
d^{2}=\left(r^{\prime}-r\right)^{2}+2 r^{\prime} r\left(1-\cos \left(\theta^{\prime}-\theta\right)\right)+2 r^{\prime} r \sin \theta^{\prime} \sin \theta\left(1-\cos \left(\phi^{\prime}-\phi\right)\right)(65)
$$


it is apparent that the integrals must be performed in the order $\phi \rightarrow \theta \rightarrow r$ and that only those stationary points that do not give an additional phase contribute to the final result.

As eigenfunctions we choose the orthogonal set

$$
\Psi_{n l m}(r \theta \phi)=R_{n l}(r) Y_{l m}(\theta) \Phi_{m}(\phi) .
$$

The transition element is

$$
\begin{aligned}
G_{n^{\prime} l^{\prime} m^{\prime}, n m l}(t)= & \int_{r_{1}}^{r_{2}} r^{\prime 2} d r^{\prime} \int_{r_{1}}^{r_{2}} r^{2} d r R_{n^{\prime} l^{\prime}}^{*}\left(r^{\prime}\right) R_{n l}(r) \\
& \times \sqrt{\frac{M}{2 \pi i \hbar t}} e^{i \frac{M\left(r^{\prime}-r\right)^{2}}{2 \hbar t}} \frac{1}{r^{\prime} r} G_{l^{\prime} m^{\prime}, l m}, \\
G_{l^{\prime} m^{\prime}, l m} & =\int_{0}^{\pi} \sin \theta^{\prime} d \theta^{\prime} \int_{0}^{\pi} \sin \theta d \theta Y_{l^{\prime} m^{\prime}}^{*}\left(\theta^{\prime}\right) Y_{l m}(\theta) \\
& \times \sqrt{\frac{M r^{\prime} r}{2 \pi i \hbar t}} e^{i \frac{M}{\hbar t} r^{\prime} r\left(1-\cos \left(\theta^{\prime}-\theta\right)\right)} \\
& \times \frac{1}{\sqrt{\sin \theta^{\prime} \sin \theta}} \int_{0}^{2 \pi} d \phi^{\prime} \int_{0}^{2 \pi} d \phi \sqrt{\frac{M r^{\prime} \sin \theta^{\prime} r \sin \theta}{2 \pi i \hbar t}} \\
& \times e^{i \frac{M}{\hbar t} r^{\prime} r \sin \theta^{\prime} \sin \theta\left(1-\cos \left(\phi^{\prime}-\phi\right)\right)} \Phi_{m^{\prime}}^{*}\left(\phi^{\prime}\right) \Phi_{m}(\phi)
\end{aligned}
$$

The $\phi^{\prime}, \phi$ integral is immediately computed as in the case of the annulus, Sec. 4. $\Phi_{m}$ must therefore satisfy the differential equation

$$
-\Phi_{m}^{\prime \prime}=\alpha_{m} \Phi_{m}, \quad \Phi_{m}(0)=\Phi_{m}(2 \pi),
$$

so that $\alpha_{m}=m^{2}$. Then $G_{l^{\prime} m^{\prime} l m}$ becomes

$$
\begin{aligned}
G_{l^{\prime} m^{\prime}, l m}= & \int_{0}^{\pi} \sqrt{\sin \theta^{\prime}} d \theta^{\prime} \int_{0}^{\pi} \sqrt{\sin \theta} d \theta Y_{l^{\prime} m^{\prime}}^{*}\left(\theta^{\prime}\right) Y_{l m}(\theta) \sqrt{\frac{M r^{\prime} r}{2 \pi i \hbar t}} \\
& \times e^{i \frac{M}{\hbar t} r^{\prime} r\left(1-\cos \left(\theta^{\prime}-\theta\right)\right)}\left(1-i \frac{\hbar t}{2 M r^{\prime} \sin \theta^{\prime} r \sin \theta}\left(m^{2}-1 / 4\right)\right) \delta_{m^{\prime} m} .
\end{aligned}
$$

The integral over $\theta^{\prime}, \theta$ can be computed in a standard way (do not forget the $\xi^{4}$ term in the cosine series) and this in turn requires that the function $A_{l m}=\sqrt{\sin \theta} Y_{l m}$ must satisfy the differential equation

$$
A_{l m}^{\prime \prime}+\frac{1}{4} A_{l m}-\frac{m^{2}-1 / 4}{\sin ^{2} \theta} A_{l m}=-\alpha_{l m} A_{l m}
$$

or, equivalently,

$$
\frac{1}{\sin \theta} \frac{\partial}{\partial \theta}\left(\sin \theta \frac{\partial}{\partial \theta}\right) Y_{l m}-\frac{m^{2}}{\sin ^{2} \theta} Y_{l m}=-\alpha_{l m} Y_{l m}
$$

with $Y_{l m}(0)=Y_{l m}(\pi)$. This is the standard equation for spherical harmonics. It is known that $\alpha_{l m}=l(l+1)$ irrespectively of the value of $m$. We obtain

$$
\begin{aligned}
G_{n^{\prime} l^{\prime} m^{\prime} n l m}= & \int_{r_{1}}^{r_{2}} r^{\prime} d r^{\prime} \int_{r_{1}}^{r_{2}} r d r R_{n^{\prime} l^{\prime}}^{*}\left(r^{\prime}\right) R_{n l}(r) \sqrt{\frac{M}{2 \pi i \hbar t}} e^{i \frac{M\left(r^{\prime}-r\right)^{2}}{2 \hbar t}} \\
& \times\left(1-i \frac{\hbar l(l+1)}{2 M r^{\prime} r} t\right) \delta_{l^{\prime} l} \delta_{m^{\prime} m} .
\end{aligned}
$$


Finally, the differential equation for $A_{n l}=r R_{n l}$ reads (here $E_{n l}=\frac{\hbar^{2} k_{n l}^{2}}{2 M}$, which is independent of $m$ )

$$
-A_{n l}^{\prime \prime}+\frac{l(l+1)}{r^{2}} A_{n l}=k_{n l}^{2} A_{n l}, \quad A_{n l}\left(r_{1}\right)=A_{n l}\left(r_{2}\right)=0,
$$

or, equivalently, in terms of $R_{n l}$, Eq. (63) . The Zeno Hamiltonian is therefore given by (61) $-(62)$.

\section{The general case}

By looking at the preceding examples one might think that the method introduced in this article is parochial and works only, for example, when the domain, besides being sufficiently regular, is also endowed with particular symmetries (regular polygons, circles, spheres and so on), that enable one to introduce coordinates with a range of integration that can be reduced to a product of intervals. In turn, this might appear as an implicit condition of separability, e.g. in the case of the three-dimensional Schrödinger equation 23]. On the contrary, as will be shown in this section, the method we propose is of general applicability.

Consider again the Hamiltonian (11) and the projection (2), $D \subset \mathbb{R}^{N}$ being a compact domain with nonempty interior and a regular boundary. The $N$-dimensional propagator (10) reads

$$
\begin{aligned}
G_{\boldsymbol{m}, \boldsymbol{n}}(t) & =\langle\boldsymbol{m}|G(t)| \boldsymbol{n}\rangle \\
& =\int_{D} d^{N} x \int_{D} d^{N} y\left(\frac{M}{2 \pi i \hbar t}\right)^{N / 2} e^{i \frac{M(\boldsymbol{x}-\boldsymbol{y})^{2}}{2 \hbar t}} \Psi_{\boldsymbol{m}}^{*}(\boldsymbol{x}) \Psi_{\boldsymbol{n}}(\boldsymbol{y})
\end{aligned}
$$

and by substituting $\boldsymbol{\xi}=\boldsymbol{y}-\boldsymbol{x}$ one gets

$$
\begin{aligned}
G_{\boldsymbol{m}, \boldsymbol{n}}(t) & =\int_{D} d^{N} x \Psi_{\boldsymbol{m}}^{*}(\boldsymbol{x}) \int_{D-\boldsymbol{x}} d^{N} \xi\left(\frac{M}{2 \pi i \hbar t}\right)^{N / 2} e^{i \frac{M \boldsymbol{\xi}^{2}}{2 \hbar t}} \Psi_{\boldsymbol{n}}(\boldsymbol{x}+\boldsymbol{\xi}) \\
& =\int_{D} d^{N} x \Psi_{\boldsymbol{m}}^{*}(\boldsymbol{x})[\text { bound }+ \text { stat }]
\end{aligned}
$$

where

$$
D-\boldsymbol{x}=\{\boldsymbol{y} \mid \boldsymbol{x}+\boldsymbol{y} \in D\} .
$$

Let us evaluate separately the two contributions in the small- $t$ limit. In order to compute the boundary term, we first observe that

$$
e^{i \lambda \boldsymbol{\xi}^{2}}=\frac{\boldsymbol{\xi} \cdot \nabla e^{i \lambda \boldsymbol{\xi}^{2}}}{2 i \lambda \boldsymbol{\xi}^{2}}
$$

and then integrate by parts $(\lambda=M / 2 \hbar t)$

$$
\begin{aligned}
\text { bound } & =\int_{D} d^{N} \xi\left(\frac{\lambda}{\pi i}\right)^{N / 2} \Psi_{\boldsymbol{n}}(\boldsymbol{x}+\boldsymbol{\xi}) \frac{\boldsymbol{\xi} \cdot \nabla e^{i \lambda \boldsymbol{\xi}^{2}}}{2 i \lambda \boldsymbol{\xi}^{2}} \\
& =\left(\frac{\lambda}{\pi i}\right)^{N / 2}\left[\int_{D} d^{N} \xi \nabla \cdot\left(\frac{\Psi_{\boldsymbol{n}}(\boldsymbol{x}+\boldsymbol{\xi}) \boldsymbol{\xi} e^{i \lambda \boldsymbol{\xi}^{2}}}{2 i \lambda \boldsymbol{\xi}^{2}}\right)\right.
\end{aligned}
$$




$$
\begin{gathered}
\left.-\int_{D} d^{N} \xi \nabla \cdot\left(\frac{\Psi_{\boldsymbol{n}}(\boldsymbol{x}+\boldsymbol{\xi}) \boldsymbol{\xi}}{\boldsymbol{\xi}^{2}}\right) \frac{\boldsymbol{\xi} \cdot \nabla e^{i \lambda \boldsymbol{\xi}^{2}}}{(2 i \lambda)^{2} \boldsymbol{\xi}^{2}}\right] \\
=\left(\frac{\lambda}{\pi i}\right)^{N / 2}\left[\oint_{\partial(D-\boldsymbol{x})} d^{N-1} S \frac{\Psi_{\boldsymbol{n}}(\boldsymbol{x}+\boldsymbol{\xi}) \boldsymbol{\xi} \cdot \hat{\boldsymbol{u}}}{\boldsymbol{\xi}^{2}} \frac{e^{i \lambda \boldsymbol{\xi}^{2}}}{2 i \lambda}\left(1+O\left(\lambda^{-1}\right)\right)\right] \\
=\left(\frac{M}{2 \pi i \hbar t}\right)^{N / 2}\left[\oint_{\partial D} d^{N-1} S \frac{\Psi_{\boldsymbol{n}}(\boldsymbol{y})(\boldsymbol{y}-\boldsymbol{x}) \cdot \hat{\boldsymbol{u}}}{(\boldsymbol{y}-\boldsymbol{x})^{2}} \frac{e^{i M(\boldsymbol{x}-\boldsymbol{y})^{2} / 2 \hbar t}}{i M / \hbar t}\right. \\
(1+O(t))],
\end{gathered}
$$

$\hat{\boldsymbol{u}}$ being the unit vector perpendicular to the boundary. In the second equality, Eq. (78) was used again in order to obtain a higher-order volume integral with the same structure as the initial one. The stationary contribution is obtained, as usual, by expanding the integrand function around $\boldsymbol{x}$

$$
\begin{aligned}
\text { stat }= & \left(\frac{M}{2 \pi i \hbar t}\right)^{N / 2} \int d^{N} \xi e^{i \lambda \boldsymbol{\xi}^{2}} \\
& \times\left(\Psi_{\boldsymbol{n}}(\boldsymbol{x})+\nabla \Psi_{\boldsymbol{n}}(\boldsymbol{x}) \cdot \boldsymbol{\xi}+\frac{1}{2 !} \partial_{i} \partial_{j} \Psi_{\boldsymbol{n}}(\boldsymbol{x}) \xi_{i} \xi_{j}+O\left(|\boldsymbol{\xi}|^{3}\right)\right) .
\end{aligned}
$$

Observe that the contributions of the linear and quadratic (with $i \neq j$ ) terms in the integral vanish due to symmetry and one is left with

$$
\text { stat }=\Psi_{\boldsymbol{n}}(\boldsymbol{x})+i \frac{t \hbar}{2 M} \triangle \Psi_{\boldsymbol{n}}(\boldsymbol{x})+O\left(t^{2}\right) .
$$

In order to obtain (11)-(12) from (176) one must require that the leading contribution in the boundary term (79) vanishes and

$$
-\frac{\hbar}{2 M} \triangle \Psi_{\boldsymbol{n}}(\boldsymbol{x})=\frac{E_{\boldsymbol{n}}}{\hbar} \Psi_{\boldsymbol{n}}(\boldsymbol{x})
$$

namely

$$
-\frac{\hbar^{2}}{2 M} \triangle \Psi_{\boldsymbol{n}}(\boldsymbol{x})=E_{\boldsymbol{n}} \Psi_{\boldsymbol{n}}(\boldsymbol{x}), \quad \text { with } \quad \Psi_{\boldsymbol{n}}(\partial D)=0 .
$$

Notice that the Schrödinger equation is obtained from the stationary contribution to the asymptotic expansion, while the Dirichlet boundary conditions are a consequence of the requirement that the boundary term (79) vanish at the lowest order in the expansion.

Let us briefly comment on the features of the method introduced. As already emphasized at the end of Section 3, this analysis, although not entirely rigorous, yields the correct result. We derived the desired properties of the propagator by requiring at the same time the validity of the Schrödinger equation and the Dirichlet (hardwall) boundary conditions for the eigenbasis of the (Zeno) Hamiltonian. We should emphasize, however, that the boundary and stationary terms are being dealt with separately. In fact, we did not consider the contribution of those boundary points that are also stationary points. Such points belong to the intersection of the boundaries of the two domains $D$ in (75), namely $\boldsymbol{x}=\boldsymbol{y} \in \partial D$, and should be analyzed with great care. A more rigorous treatment can be given, in which the contribution of the integral 
(76) is uniformly estimated: this analysis requires a different evaluation of the boundary terms and will be presented elsewhere.

The introduction of a potential [6] is not difficult to deal with if the detailed features of the convergence (10)-(11) are not worked out. Much additional care is required at a deeper mathematical level, when the self adjointness of the Hamiltonian is called into question and must be explicitly proved. If additional rigorous results [2, 16, 17, 21, are taken into account and, by an educated guess, extended to the case of a sufficiently regular potential, one is tempted to assume that the procedure sketched above is valid in general and the Zeno dynamics governed by a self-adjoint generator (and a unitary group). The situation may clearly become more complicated when the potential is singular and/or the projected spatial region (or its boundary) lacks the required regularity.

\section{Zeno dynamics in Heisenberg picture}

In this section we would like to consider the Zeno dynamics in the framework of the Heisenberg picture. The following discussion must be considered preliminary: additional details and a broader picture will be given in a forthcoming paper. An interesting and natural question concerns the algebra of observables after the projection. This is not a simple problem. One can assume that to a given observable $\mathcal{O}$ before the Zeno projection procedure there corresponds the observable $P \mathcal{O} P$ in the projected space:

$$
\mathcal{O} \Rightarrow P \mathcal{O} P
$$

For example, if one starts in $\mathbb{R}$ and projects over a finite interval $P=\chi_{I}(x)$ ( $I$ being an interval of $\mathbb{R}$ ), the momentum and position operators become

$$
\begin{aligned}
& p \Rightarrow P p P=\left\{\begin{array}{cc}
i \partial_{x} & \text { for } x \in I \\
0 & \text { otherwise }
\end{array},\right. \\
& x \Rightarrow P x P=\left\{\begin{array}{ll}
x & \text { for } x \in I \\
0 & \text { otherwise }
\end{array} .\right.
\end{aligned}
$$

In this respect it is easy to see that the correspondence (84) is not an algebra homomorphism. However, if we redefine a new associative product in the algebra of operators, by setting

$$
A * B \equiv A P B
$$

with this new product the previous correspondence (84) becomes an algebra homomorphism [24]. Notice also that the new (projected) algebra acquires a unity operator $P$.

Notice that in general the evolution will not be an automorphism of the new product. However, it will respect the product to order $O(t / N)$ and induce, in the limit, a Zeno dynamics on the projected algebra, i.e. on the image of the projection. The evolution will be trivially an automorphism when it commutes with $P$ and is therefore compatible with the new product without any approximation. For instance, this would 
be the case if we take as Hamiltonian the square of the angular momentum in the case of the annulus (Sec. 4).

In general one has to modify the associative product in such a way that the "deviation" of $U(t / N)$ from being an automorphism is of order $o(t / N)$, so that in the limit $U_{Z}(t)$ will be an automorphism of the new associative product adapted to the constraint. In other words, the sequence of evolution operators

$$
V_{N}(t)=G(t / N)^{N}=(P U(t / N) P)^{N}
$$

yielding the Zeno limit (3), is mirrored at the level of the algebra by the following sequence of deformed associative products

$$
A *_{N} B \equiv A P_{N} B
$$

where $P_{N}$ is a smooth positive operator with $0 \leq P_{N} \leq 1$ and $P_{N} P=P P_{N}=P$. For any $N, P_{N}$ forms together with $Q_{N}=1-P_{N}$ a positive operator valued measure, yielding a resolution of the identity, i.e. $P_{N}+Q_{N}=1$, which approximates the orthogonal resolution $P+Q=1$, in the sense that

$$
P_{N} \psi=P \psi+O(1 / N), \quad \forall \psi \in L^{2}\left(\mathbb{R}^{N}\right) .
$$

For any $N$ the evolution $V_{N}(t)$ is an automorphism of the product $*_{N}$ and in the limit $N \rightarrow \infty$ we get the desired result (87).

Observe that, for unbounded operators, (84) does not necessarily yield self-adjoint operators: for example, after the Zeno procedure, the momentum $p$ would act on functions that vanish on the boundary of $I$ and would have deficiencies $\langle 1,1\rangle$, see [5]. On the other hand the Zeno Hamiltonian (6) is self adjoint. However, it would be arbitrary to require a similar property for every observable in the algebra. We shall analyze this issue in greater detail in a future article. In general, the lack of self-adjointness of the operators representing the "observables" of the system in the projected subspace might be related to the incompleteness of the corresponding classical field [25, 5].

\section{Projections onto lower dimensional regions: constraints}

In all the situations considered so far, the projected domain always has the same dimensionality of the original space $\left(\mathbb{R}^{n}\right)$. [Remember that, after Eq. (11), we required the projected domain $D$ to have a nonempty interior.] However, it is interesting to ask what would happen if one would project onto a domain $D^{\prime}$ of lower dimensionality. This is clearly a more delicate problem, as one necessarily has to face the presence of divergences. It goes without saying that these divergences must be ascribed to the lower dimensionality of the projected domain and not directly to the convergence features of the Zeno propagator [26]. Our problem is to understand how these divergences can be cured. One way to tackle this problem is to start from a projection onto a domain $D \subset \mathbb{R}^{n}$ and then take the limit $D \rightarrow D^{\prime} \subset \mathbb{R}^{n-1}$, with a Hilbert space (Zeno subspace) $L^{2}\left(D^{\prime}\right)$. 
The content of this section is preliminary. We shall only sketch the main ideas and postpone a thorough analysis to a forthcoming paper, in which the physical meaning of the divergences will be spelled out in greater details.

\subsection{From the rectangle to the interval}

Let us first look at the case of the rectangle, investigated in Sec. 3, and let $b \rightarrow 0$. We first notice that in order to get a sensible result one must first perform the Zeno limit $N \rightarrow \infty$ and then let $b \rightarrow 0$. In particular one must require

$$
\delta t=t / N \ll \hbar / E_{m}=\frac{2 M b^{2}}{\hbar m^{2}},
$$

which has an appealing physical meaning: the time during which the particle evolves freely between two projections must be small enough so that the particle remains well within the rectangle of width $b$. In practice, one must first set $m<m^{*}$, for some $m^{*}$, in order to obtain a sensible result and finally let $m^{*}$ become arbitrarily large. The order in which the two limits $(N \rightarrow \infty$ and $b \rightarrow 0)$ are taken is relevant and significant from a physical perspective: one must first make sure that the wave function does not "leak" out of the projected (Zeno) region and then let this region "shrink" into a domain of lower dimensionality.

However, even if one follows the correct procedure (i.e., first $N \rightarrow \infty$ and then $b \rightarrow 0$ ) one still gets divergences in the phases, since

$$
E_{m}=\frac{\hbar^{2} \pi^{2} m^{2}}{2 M b^{2}} \rightarrow \infty \quad \text { for } \quad b \rightarrow 0 .
$$

Notice also that since the energy differences between different $m$ states diverge, a superselection rule arises. Different subspaces, labeled by different values of the quantum number $m$, remain separated (at least for low-energy processes with energies $\left.E \ll \hbar^{2} / M b^{2}\right)$. This is also physically revealing.

On the basis of the above insights, we therefore propose to perform the limit $b \rightarrow 0$ by choosing a particular eigenstate $\phi_{m}(y)$ and considering the reduced evolution

$$
\widetilde{U}_{Z}(t)=e^{i E_{m} t / \hbar}\left\langle m\left|U_{Z}(t)\right| m\right\rangle,
$$

which operates only on the $x$ degree of freedom. Physically, this corresponds to the choice of a particular value of the superselection charge. Thus, the reduced propagator reads

$$
\begin{aligned}
G\left(x^{\prime}, x ; t\right)=\left\langle x^{\prime}\left|\widetilde{U}_{Z}(t)\right| x\right\rangle & =e^{i E_{m} t / \hbar}\left\langle m ; x^{\prime}\left|U_{Z}(t)\right| m ; x\right\rangle \\
& =\sum_{n} e^{-i E_{n} t / \hbar} \psi_{n}\left(x^{\prime}\right) \psi_{n}^{*}(x),
\end{aligned}
$$

where $\psi_{n}$ are the eigenfunctions of the Dirichlet problem (32), and one gets

$$
\widetilde{H}_{Z}=-\frac{\hbar^{2} \partial_{x}^{2}}{2 M}, \quad \text { with Dirichlet b.c. }
$$

This is just the free particle on the interval $[0, a]$, as expected. Not only can the divergence be cured, it also yields the desired result. 
The framework explained in this particular example works also in more complicated circumstances. In particular, it is important to understand in which order the two limits must be computed: first one makes sure that the Zeno mechanism works efficaciously, then takes the desired limit on the domain. We consider here two other simple situations.

\subsection{From the annulus to the circle}

Let us now look at the annulus, investigated in Sec. 4. We would like to recover the evolution of a particle on a circle by considering the $\delta r \rightarrow 0$ limit, while keeping $r_{1}=r_{2}=R$ constant. Once again, as in Sec. 8.1, we have to face some divergences. By taking the limit one finds the approximate eigenfunctions of Eq. (41)

$$
\psi_{n l}(r) \simeq \psi_{n}(r)=\sqrt{\frac{2}{R \delta r}} \sin \left(\frac{n \pi}{\delta r}(r-R)\right)
$$

and the energies

$$
E_{n l} \simeq E_{n}+E_{l}=\frac{\hbar^{2}}{2 M} \frac{n^{2} \pi^{2}}{\delta r^{2}}+\frac{\hbar^{2}}{2 M R^{2}}\left(l^{2}-\frac{1}{4}\right) .
$$

Again one finds a diverging energy which must be regularized. However a second (finite) term appears $\left(-\hbar^{2} / 8 M R^{2}\right)$ [27] which is not present in the usual circle quantization. We notice that different quantization methods yield different results [28].

The reduced propagator on the remaining degree of freedom $\theta$ is just

$$
G\left(\theta^{\prime}, \theta ; t\right)=e^{i \frac{\hbar^{2}}{2 M} \frac{n^{2} \pi^{2}}{\delta r^{2}}}\left\langle n, \theta^{\prime}\left|U_{Z}(t)\right| n, \theta\right\rangle=\sum_{l} e^{-i E_{l} t / \hbar} \phi_{l}^{*}\left(\theta^{\prime}\right) \phi_{l}(\theta),
$$

which is what one expected.

\subsection{From the shell to the sphere}

Finally, we reconsider the spherical shell of Sec. 5 and take the limit $\delta r \rightarrow 0$, while keeping $r_{1}=r_{2}=R$ constant, like in Sec. 8.2. This yields the energies

$$
E_{n l} \simeq \frac{\hbar^{2} n^{2} \pi^{2}}{2 m \delta r^{2}}+\frac{\hbar^{2} l(l+1)}{2 M R^{2}}
$$

and following the same regularization procedure as before we find

$$
\begin{aligned}
G\left(\theta^{\prime}, \phi^{\prime}, \theta, \phi ; t\right) & =e^{i \frac{\hbar \pi^{2} n^{2}}{2 M \delta r^{2}} t}\left\langle n ; \theta^{\prime}, \phi^{\prime}\left|U_{Z}(t)\right| n ; \theta, \phi\right\rangle \\
& =\sum_{l m} e^{-i \frac{\hbar l(l+1)}{2 M R^{2}} t} Y_{l m}\left(\theta^{\prime}\right) \Phi_{m}\left(\phi^{\prime}\right) Y_{l m}^{*}(\theta) \Phi_{m}^{*}(\phi),
\end{aligned}
$$

which is the usual propagator on a sphere of radius $R$, whose Hamiltonian is

$$
\tilde{H}_{Z}=\frac{\boldsymbol{L}^{2}}{2 M R^{2}}=-\frac{\hbar^{2}}{2 M R^{2}}\left(\frac{1}{\sin \theta} \partial_{\theta}\left(\sin \theta \partial_{\theta}\right)+\frac{1}{\sin ^{2} \theta} \partial_{\phi}^{2}\right) \text {. }
$$




\section{Concluding remarks on potential applications}

We have investigated the quantum Zeno dynamics, when a free system undergoes frequent measurements that ascertain whether it is within a sufficiently regular spatial region. The evolution in the projected (Zeno) subspace is unitary and the generator of the Zeno dynamics is the Hamiltonian with hard-wall (Dirichlet) boundary conditions. In general, this procedure leads to the formation of the "Zeno subspaces" [7], on whose boundaries the wave function must vanish (Dirichlet): this is the ultimate reason for the absence of amplitude (and probability) leakage between "adjacent" subspaces.

Quantum computation [29] is one of the most promising fields of potential application of the QZE. Interactions with the environment deteriorate the purity of quantum states and represent a very serious obstacle against the preservation of quantum superpositions and entanglement over long periods of time. It is therefore of great interest to endeavor to understand whether decoherence can be controlled and eventually halted [30]: in this context, novel techniques hinging upon the quantum Zeno effect are of interest. Besides the use of quantum error correcting codes [31, the engineering of "decoherence-free" subspaces is also recently being considered and widely investigated 32. Some mechanisms are actually being proposed, based on the so-called "bang-bang"

evolutions and their generalization, quantum dynamical decoupling [33]. Although "bang-bang" techniques in classical control theory are know to engineers since long ago 34, their introduction as a quantum control and their unification with the basic ideas underlying the quantum Zeno effect are quite recent [35. In particular, the decoherencefree subspaces are the dynamically generated quantum Zeno subspaces [7] within which the dynamics is far from being trivial, as has been discussed in this article. It is also worth noticing that the range of applicability of these ideas is wide, as frequent interruptions and continuous coupling [36] can yield similar physical effects. This is not entirely surprising [37, in view of Wigner's notion of "spectral decomposition" 38. However, when one considers applications of the Zeno dynamics in the context of decoherence-free subspaces, one must remember that if the measurement is not very frequent, the quantum evolution yields the so-called "inverse" or "anti" Zeno effect, by which transitions out of the decoherence-free subspace is accelerated [8].

In conclusion, it is interesting to notice that an issue that was considered as purely academic until a few years ago, has been first experimentally demonstrated and is now being considered as a possible strategy to combat decoherence, with interesting spinoffs and very practical applications.

\section{References}

[1] von Neumann J 1955 Mathematical Foundation of Quantum Mechanics (Princeton: Princeton University Press)

Beskow A and Nilsson J 1967 Arkiv für Fysik 34561

Khalfin L A 1968 JETP Letters 865

[2] Misra B and Sudarshan E C G 1977 J. Math. Phys. 18756 
[3] Home D and Whitaker M A B 1997 Ann. Phys. 258237

Facchi P and Pascazio S 2001 Progress in Optics Wolf E (ed) (Amsterdam: Elsevier) 42147

[4] Schwinger J 1959 Proc. Natl. Acad. Sci. U.S. 451552

1970 Quantum Kinetics and Dynamics (New York: Benjamin)

[5] Facchi P, Gorini V, Marmo G, Pascazio S and Sudarshan E C G 2000 Phys. Lett. A 27512

[6] Facchi P, Pascazio S, Scardicchio A and Schulman L S 2002 Phys. Rev. A 65012108

[7] Facchi P and Pascazio S 2002 Phys. Rev. Lett. 89080401

_ "Quantum Zeno subspaces and dynamical superselection rules" 2003 in Proceedings of the 22nd Solvay Conference (Singapore: World Scientific) 251

[8] Lane A M 1983 Phys. Lett. A 99359

Schieve W C, Horwitz L P and Levitan J 1989 Phys. Lett. A 136264

Kaulakys B and Gontis V 1997 Phys. Rev. A 561131

Thun K and Peřina J 1998 Phys. Lett. A 249363

Facchi P and Pascazio S 2000 Phys. Rev. A 62023804

Elattari B and Gurvitz S A 2000 Phys. Rev. A 62032102

Kofman A G and Kurizki G 2000 Nature 405546

Facchi P, Nakazato H and Pascazio S 2001 Phys. Rev. Lett. 862699

Koshino K and Shimizu A 2003 Phys. Rev. A 67042101

[9] Itano W M, Heinzen D J, Bolinger J J and Wineland D J 1990 Phys. Rev. A 412295

[10] Wilkinson S R, Bharucha C F, Fischer M C, Madison K W, Morrow P R, Niu Q, Sundaram B and Raizen M G 1997 Nature $\mathbf{3 8 7} 575$

[11] Fischer M C, Gutiérrez-Medina B and Raizen M G 2001 Phys. Rev. Lett. 87040402

[12] Balzer C, Huesmann R, Neuhauser W and Toschek P E 2000 Opt. Comm. 180115

[13] Valanju P, Sudarshan E C G and Chiu C B 1980 Phys. Rev. D 211304

[14] Cook R J 1988 Phys. Scr. T 2149

[15] Peres A and Ron A 1990 Phys. Rev. A 425720

Itano W H, Heinzen D J, Bollinger J J and Wineland D J 1991 Phys. Rev. A 435168

Inagaki S, Namiki M and Tajiri T 1992 Phys. Lett. A 1665

Pascazio S, Namiki M, Badurek G and Rauch H 1993 Phys. Lett. A 179155

Blanchard Ph and Jadczyk A 1993 Phys. Lett. A 183272

Altenmüller T P and Schenzle A 1994 Phys. Rev. A 492016

Berry M 1995 Fundamental Problems in Quantum Theory Greenberger D M and Zeilinger A (ed)

(New York: Ann. N.Y. Acad. Sci.) 755303

Beige A and Hegerfeldt G 1996 Phys. Rev. A 5353

Luis A and Peřina J 1996 Phys. Rev. Lett. 764340

[16] Friedman C N 1972 Indiana Univ. Math. J. 211001

[17] Gustafson K 1983 "Irreversibility questions in chemistry, quantum-counting, and time-delay" in Energy storage and redistribution in molecules, Hinze J (ed) (Plenum) and refs. [10,12] therein

[18] Misra B and Antoniou A 2003 "Quantum Zeno effect" in Proceedings of the 22nd Solvay Conference (Singapore: World Scientific) 233

[19] Gustafson K 2003 "A Zeno story" Preprint quant-ph/0203032

[20] Schmidt A U 2002 J. Phys. A: Math. Gen. 357817

2003 J. Phys. A: Math. Gen. 361135

[21] Exner P and Ichinose T 2003 "Product formula related to quantum Zeno dynamics" Preprint math-ph/0302060

[22] Sommerfeld A 1949 Partial differential equation in physics (New York: Academic Press) p 168

[23] Pauling L and Wilson E B 1935 Introduction to quantum mechanics (Singapore: McGraw-Hill) 443

Eisenhart L P 1934 Phys. Rev. 45428

[24] Man'ko V I, Marmo G, Zaccaria F and Sudarshan E C G 1997 Int. J. Mod. Phys. B 111281

Carinena J F, Grabowski J and Marmo G 2000 Int. J. Mod. Phys. A 154797 
[25] Nelson E 1969 Topics in Dynamics I: Flows, Mathematica Notes (Princeton: Princeton University Press) p 114

Zhu C and Klauder J R 1993 Am. J. Phys. 61605

[26] Froese R and Herbst I 2001 Commun. Math. Phys. 220489 and references therein

[27] Goldstone J and Jaffe R L 1992 Phys. Rev. B 4514100

[28] Scardicchio A 2002 Phys. Lett. A 3007

[29] Galindo A and Martin-Delgado M A 2002 Rev. Mod. Phys. 74347

Bouwmeester D, Ekert A and Zeilinger A (ed) 2000 The Physics of Quantum Information (Berlin: Springer)

Nielsen M A and Chuang I L 2000 Quantum Computation and Quantum Information (Cambridge: Cambridge University Press)

[30] Kofman A G and Kurizki G 2002 Phys. Rev. Lett. 87270405

Calarco T, Datta A, Fedichev P, Pazy E and Zoller P 2003 Phys. Rev. A 68012310

Tasaki S, Tokuse A, Facchi P and Pascazio S 2004 Int. J. Quant. Chem. in print, Preprint quant-ph/0210129

[31] Shor P W 1995 Phys. Rev. A 52, 2493

Calderbank A R and Shor P W 1996 Phys. Rev. A 541098

Steane A 1996 Proc. R. Soc. London A 4522551

-1996 Phys. Rev. Lett. 77793

For a review, see Preskill J 1999 in Introduction to Quantum Computation and Information Lo H K, Popescu S and Spiller T P (ed) (Singapore: World Scientific)

[32] Palma G M, Suominen K A and Ekert A K 1996 Proc. R. Soc. Lond. A 452567

Duan L M and Guo G C 1997 Phys. Rev. Lett. 791953

Zanardi P and Rasetti M 1997 Phys. Rev. Lett. 793306

Lidar D A, Chuang I L and Whaley K B 1998 Phys. Rev. Lett. 812594

Knill E, Laflamme R and Viola L 2000 Phys. Rev. Lett. 842525

For a review, see Lidar D A and Whaley K B 2003 "Decoherence-Free Subspaces and Subsystems" Preprint quant-ph/0301032

[33] Viola L and Lloyd S 1998 Phys. Rev. A 582733

Viola L, Knill E and Lloyd S 1999 Phys. Rev. Lett. 822417

-1999 Phys. Rev. Lett. 834888

2000 Phys. Rev. Lett. 853520

Zanardi P 1999 Phys. Lett. A 25877

Vitali D and Tombesi P 1999 Phys. Rev. A 594178

2001 Phys. Rev. A 65012305

Uchiyama C and Aihara M 2002 Phys. Rev. A 66032313

Byrd M S and Lidar D A 2002 Quantum Information Processing 119

2003 Phys. Rev. A 67012324

[34] Jurdjevic V 1997 Geometric control theory (Cambridge: Cambridge University Press)

[35] Facchi P, Lidar D A and Pascazio S 2004 Phys. Rev. A 69 0323XX

[36] Simonius M 1978 Phys. Rev. Lett. 40980

Peres A 1980 Am. J. Phys. 48931

Harris R A and Stodolsky L 1982 Phys. Lett. B 116464

Venugopalan A and Ghosh R 1995 Phys. Lett. A 20411

Plenio M P, Knight P L and Thompson R C 1996 Opt. Comm. 123278

Berry M V and Klein S 1996 J. Mod. Opt. 43165

Mihokova E, Pascazio S and Schulman L S 1997 Phys. Rev. A 5625

Luis A and Sánchez-Soto L L 1998 Phys. Rev. A 57781

Schulman L S 1998 Phys. Rev. A 571509

Thun K and Peřina J 1998 Phys. Lett. A 249363

Panov A D 1999 Phys. Lett. A 260441 
Řeháček J, Peřina J, Facchi P, Pascazio S and Mišta L 2000 Phys. Rev. A 62013804

Facchi P and Pascazio S 2000 Phys. Rev. A 62023804

Militello B, Messina A and Napoli A 2001 Phys. Lett. A 286369

Luis A 2001 Phys. Rev. A 64032104

[37] Petrosky T, Tasaki S and Prigogine I 1990 Phys. Lett. A 151109

-1991 Physica A $\mathbf{1 7 0} 306$

Pascazio S and Namiki M 1994 Phys. Rev. A 504582

[38] Wigner E P 1963 Am. J. Phys. 316 